\title{
Growth differentiation factor 15 predicts poor prognosis in patients with heart failure and reduced ejection fraction and anemia: results from RED-HF
}

\author{
Thor Ueland ${ }^{1,4,8}$ (D) Lars Gullestad $^{2,4,5,6} \cdot$ Lei Kou $^{9} \cdot$ James B. Young ${ }^{9} \cdot$ Marc A. Pfeffer $^{10}$. Dirk Jan van Veldhuisen ${ }^{13}$. \\ Karl Swedberg ${ }^{14,15} \cdot$ John J. V. Mcmurray ${ }^{16}$. Akshay S. Desai ${ }^{10} \cdot$ Inderjit S. Anand $^{11,12} \cdot$ Pål Aukrust $^{1,3,4,7,8}$
}

Received: 18 August 2020 / Accepted: 15 September 2021 / Published online: 5 October 2021

(c) The Author(s) 2021

\begin{abstract}
Aims We aimed to assess the value of GDF-15, a stress-responsive cytokine, in predicting clinical outcomes in patients with heart failure (HF) with reduced ejection fraction (HFrEF) and anemia

Methods and results Serum GDF-15 was assessed in $1582 \mathrm{HFrEF}$ and mild-to-moderate anemia patients who where followed for 28 months in the Reduction of Events by Darbepoetin alfa in Heart Failure (RED-HF) trial, an overall neutral RCT evaluating the effect darbepoetin alfa on clinical outcomes in patients with systolic heart failure and mild-to-moderate anemia. Association between baseline and change in GDF-15 during 6 months follow-up and the primary composite outcome of all-cause death or HF hospitalization were evaluated in multivariable Cox-models adjusted for conventional clinical and biochemical risk factors. The adjusted risk for the primary outcome increased with (i) successive tertiles of baseline GDF15 (tertile 3 HR 1.56 [1.23-1.98] $p<0.001$ ) as well as with (ii) a 15\% increase in GDF-15 levels over 6 months of followup (HR 1.68 [1.38-2.06] $p<0.001$ ). Addition of change in GDF-15 to the fully adjusted model improved the C-statistics $(p<0.001)$. No interaction between treatment and baseline or change in GDF-15 on outcome was observed. GDF-15 was inversely associated with several indices of anemia and correlated positively with ferritin.

Conclusions In patients with HF and anemia, both higher baseline serum GDF-15 levels and an increase in GDF-15 during follow-up, were associated with worse clinical outcomes. GDF-15 did not identify subgroups of patients who might benefit from correction of anemia but was associated with several indices of anemia and iron status in the HF patients.
\end{abstract}

\section{Graphic abstract}

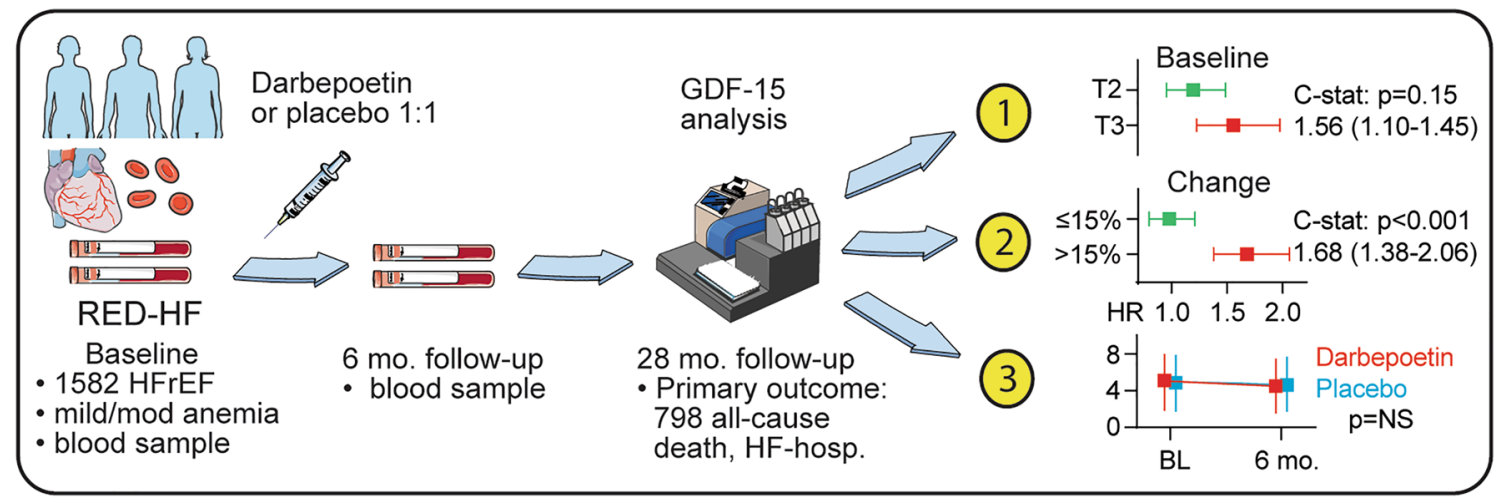

Keywords GDF-15 · Anemia · Heart failure · Prognosis

Thor Ueland

thor.ueland@medisin.uio.no

Extended author information available on the last page of the article 


\section{Introduction}

Anemia is common in patients with heart failure (HF) and is associated with a high incidence of hospitalization and death [1-3]. The cause of anemia in patients with HF is often unknown, but may be related to iron deficiency or an absolute or relative deficiency of, or resistance to, erythropoietin as well as fluid retention [2-5]. Anemia in HF patients is associated with impaired renal function, potentially causing impaired erythropoietin production, and patients with HF often have systemic inflammation, which may lead to bone marrow suppression $[2,3]$.

Growth differentiation factor-15 (GDF-15) is a stressresponsive cytokine that is activated during inflammation and tissue remodeling The $\mathrm{N}$-terminal propeptide is secreted upon proteolytic cleavage of the precursor protein as a disulfide-linked dimer with a molecular mass of $\sim 28 \mathrm{kDa}$ [6]. GDF-15 is expressed in cardiac tissue in patients with myocardial infarction [7] and in experimental models of pressure overload and cardiac hypertrophy, potentially contributing to myocardial remodeling [7, 8]. In clinical HF, serum or plasma levels of GDF-15 are increased and correlate with clinical and biochemical markers of disease severity [9]. Furthermore, high levels of GDF-15 are reported in patients with HF and anemia, correlated with iron status [10]. It has been suggested that GDF-15 may influence erythropoiesis by suppressing hepcidin expression, a major regulator of iron status [11]. However, the effect of GDF-1 on erythropoiesis is still unclear, and the suppressive effects of GDF-15 on hepcidin were tested in a very different setting (in vitro experiments in hepatocytes) than in HF patients [11]. Several studies suggest an association between high GDF-15 and adverse outcome in HF [12-15]. In the large Valsartan Heart Failure Trial (Val-HeFT, $n=1734$ ), baseline GDF15 was independently associated with mortality even after adjusting for multiple clinical and biochemical prognostic variables including BNP, $h s$-CRP, and $h s$-Troponin [16].

Personalized medicine is receiving increasing attention and there is a need to evaluate how promising biomarkers perform in more homogenous populations and to identify if they can predict a beneficial response to targeted therapy. Whereas use of the erythropoiesis-stimulating agent Darbepoetin was not associated with improvement in clinical outcomes in the overall population in REDHF (Reduction of Events by Darbepoetin alfa in Heart Failure) trial [17], a benefit of correcting anemia in some subgroup of patients cannot be ruled out. Because GDF-15 is involved in inflammation and remodeling in cardiac and extracardiac tissues and may be related to erythropoiesis, we hypothesized that plasma concentration of GDF15 may provide prognostic information in patients with
HFrEF and anemia and identify patients who may benefit from darbepoetin alfa treatment. This hypothesis was tested in 1582 patients from the RED-HF trial who were followed for 28 months and with a primary outcome of the composite of death from any cause or first hospitalization for worsening of HF.

\section{Materials and methods}

\section{Patients and study procedures}

The study design and baseline characteristics of the REDHF trial have been reported in detail previously $[18,19]$. Patients were eligible for the study if they had the New York Heart Association (NYHA) functional class II-IV; HFrEF and left ventricular EF (LVEF) $\leq 40 \%$; a hemoglobin level of 9.0-12.0 g per deciliter and were receiving guidelinerecommended HF therapy. Patients were randomly assigned in a 1:1 ratio to receive either darbepoetin alfa or placebo. The study drug was administered subcutaneously, with doses adjusted according to hemoglobin level, which was measured in a blinded fashion.

\section{Study outcomes and definitions}

The primary predefined outcome was a composite of death from any cause or first hospitalization for worsening of HF. The prespecified adjudicated secondary outcomes were (1) composite of death from cardiovascular (CV) causes or first hospitalization for worsening of HF, (2) death from any cause, and (3) CV death. Details on the definition and adjudication of all outcomes, with specific causes of $\mathrm{CV}$ death, have been described previously [17].

\section{Unresponsiveness to darbepoetin alfa}

The hematopoietic response to DA was assessed as the percentage change in hemoglobin level between baseline and week 5 (after the 2 weight-based doses of DA) as previously reported [20]. Patients in the lowest quartile did not respond at all to DA and were considered non-responders, whereas subjects in the upper three quartiles were considered responders.

\section{Blood sampling and biochemical analyses}

At randomization and 6-month follow-up, fasting venous blood was collected and serum and plasma were separated and stored at $-80^{\circ} \mathrm{C}$ until thawing for assay. All blood samples were non-fasting and all biomarkers, except for GDF-15, were measured at a central laboratory including $\mathrm{N}$-terminal pro-brain natriuretic peptide (NT-proBNP), high 
sensitivity assays for C-reactive protein (hsCRP), troponin $\mathrm{T}$ (hsTnT), serum iron, transferrin saturation and ferritin (measured as light-chain ferritin) (Medical Research Laboratories, Zaventem, Belgium). Plasma concentration of GDF15 was analyzed by enzyme immunoassays from R\&D Systems (Minneapolis, MN, USA) with intra- and inter-assay coefficients of variation $<10 \%$. All samples were thawed an equal number of times ( $<2$ times). The analyses were performed in a 384-format using the combination of a SELMA (Jena, Germany) pipetting robot and a BioTek (Winooski, VT, USA) dispenser/washer. Absorption was read at $450 \mathrm{~nm}$ with wavelength correction set to $540 \mathrm{~nm}$ using an ELISA plate reader (Bio-Rad, Hercules, CA, USA).

\section{Statistical analysis}

See Supplemental File for a full description of statistics. Kaplan-Meier curves were constructed to visualize and evaluate (log rank test) differences in survival. A restricted cubic spline analysis with three knots was undertaken on the primary outcome to assess linearity of risk. Survival analyses were performed using the Cox proportional hazard regression models to estimate hazard ratios (HRs) and 95\% confidence intervals (CIs) for GDF-15 as a log-transformed continuous variables at baseline, which included age, gender, NYHA class, hospitalization for HF within 6 months, log serum creatinine, LVEF, etiology, body mass index (BMI), left bundle-branch block, history of atrial fibrillation or flutter, systolic blood pressure) at step one, log-transformed serum concentrations of NT-proBNP, hsTnT and hsCRP at step two. For the analysis of changes in GDF-15 concentrations from baseline to 6-month follow-up, a $15 \%$ relative change was used as cutoff, which is consistent with other studies [21]. Tertile changes were also assessed. A two-sided $p$ value $<0.05$ was considered to be significant. All statistical analyses were performed with the use of SAS software, version 9.2.

\section{Results}

\section{Baseline correlates of GDF-15}

Of the 2278 patients enrolled in the RED-HF study, baseline measurement of GDF-15 was available for $1582(69 \%)$. The median plasma level of GDF-15 at baseline in the overall population was $4170 \mathrm{ng} / \mathrm{L}$ (IQR $2669-6272 \mathrm{ng} / \mathrm{L})$. There were no differences in demographics comparing participants in the biomarker substudy population with the main RED-HF population and few differences between the treatment groups except modestly higher NYHA class and platelet count in patients receiving Darbepoetin alfa (Supplemental Table 1).
Table 1 shows the demographic and clinical characteristics according to tertiles of GDF-15. Elevated GDF-15 was associated with multiple characteristics indicating more severe HF (and worse outcomes) such as higher age, male sex, duration of disease, prevalence of diabetes, MI during the past 6 months, atrial fibrillation/flutter, poor kidney function, higher TnT and NT-proBNP levels and relevant for this population, lower iron, hemoglobin and transferrin saturation. As shown in Supplemental Table 2, stepwise linear regression identified lower hemoglobin as an independent predictor of GDF-15.

\section{Association of baseline GDF-15 levels and outcomes}

During a mean follow-up of 28 months (range 0.03-72.4 months), 798 patients reached a primary endpoint, 716 patients reached the secondary endpoints, while 649 patients died and of these, 543 due to CV causes. Cubic spline analysis revealed an increase in risk of the primary endpoint with increasing GDF-15 (Fig. 1A) and this was mirrored by the Kaplan-Meier analysis (Fig. 1B) indicating a stepwise increase in the risk for the primary outcome with increasing tertiles (3rd tertile HR 4.05 [3.25-4.90]) relative to the lowest tertile in cox regression (adjusted for randomization) with a similar pattern for the other endpoints (Table 2). In multivariable analysis adjusting for pre-specified clinical variables (as outlined in statistical methods), the association between GDF-15 and outcome, evaluated according to tertiles and as a continuous variable, while attenuated, remained significantly associated with all end points with HR's ranging from 2.47 to 2.62 for tertile 3 (all $p<0.001$ ) (Step 1, Table 2). Addition of NT-proBNP, TnT and CRP to the multivariable models, attenuated the predictive value of GDF-15, but it remained significantly associated with all outcomes with HRs around 1.5 (Step 2, Table 2). Adding GDF-15 to the fully adjusted model did not improve the C-statistics; however, a significant effect on NRI was observed for all endpoints except cardiovascular mortality (Table 2).

\section{Comparison of the prognostic value of GDF-15 with NT-proBNP and hs-TnT}

As shown in Supplemental Figure 1, GDF-15, NT-proBNP and $\mathrm{TnT}$ all contribute to a model without any other biomarker, with the biggest gain by NT-proBNP followed by TnT and GDF-15. Similarly, the largest decrease in c statistic was seen when NT-proBNP is subtracted from the full model including all biomarkers. For all-cause mortality, all markers contribute to a similar degree. 
Table 1 Baseline characteristics of the patients by GDF-15 tertiles

\begin{tabular}{|c|c|c|c|c|}
\hline $\begin{array}{l}\text { Characteristic } \\
\text { GDF-15 range (ng/L) }\end{array}$ & $\begin{array}{l}\mathrm{T} 1, n=529 \\
(500-3121)\end{array}$ & $\begin{array}{l}\mathrm{T} 2, n=526 \\
(3122-5394)\end{array}$ & $\begin{array}{l}\mathrm{T} 3, n=527 \\
(5399-20,480)\end{array}$ & $p$ value \\
\hline Age, yrs & $65 \pm 13$ & $71 \pm 11$ & $73 \pm 10$ & $<0.001$ \\
\hline Female sex & 64 & 39 & 28 & $<0.001$ \\
\hline Race (white/black) & $59 / 14$ & $68 / 8$ & $72 / 7$ & $<0.001$ \\
\hline BMI (SD) $\mathrm{kg} / \mathrm{m}^{2}$ & $27.6 \pm 5.9$ & $27.2 \pm 5.7$ & $26.4 \pm 5.5$ & $<0.001$ \\
\hline NYHA (III or IV) & 64 & 67 & 68 & 0.304 \\
\hline Ischemic HF & 60 & 75 & 80 & $<0.001$ \\
\hline Duration HF, yrs & $5.0 \pm 5.1$ & $5.3 \pm 5.4$ & $5.8 \pm 5.7$ & 0.012 \\
\hline LVEF, $\%$ & $31.1 \pm 6.4$ & $30.2 \pm 6.8$ & $29.5 \pm 7.3$ & 0.005 \\
\hline \multicolumn{5}{|l|}{ Medical history } \\
\hline Hypertension & 74 & 74 & 74 & 0.805 \\
\hline Diabetes & 32 & 48 & 54 & $<0.001$ \\
\hline Atrial fibrillation or flutter & 20 & 30 & 45 & $<0.001$ \\
\hline MI last 6 mo & 28 & 35 & 47 & $<0.001$ \\
\hline \multicolumn{5}{|l|}{ Medication } \\
\hline $\mathrm{ACE}$ or $\mathrm{ARB}$ & 95 & 91 & 84 & $<0.001$ \\
\hline Beta-blocker & 85 & 86 & 85 & 0.980 \\
\hline Diuretic & 87 & 91 & 96 & $<0.001$ \\
\hline Systolic BP & $123 \pm 17$ & $120 \pm 18$ & $117 \pm 19$ & $<0.001$ \\
\hline Heart rate, b.p.m. & $73 \pm 11$ & $71 \pm 12$ & $73 \pm 12$ & 0.044 \\
\hline \multicolumn{5}{|l|}{ Biochemistry } \\
\hline Creatinine, $\mathrm{mg} / \mathrm{dL}$ & $1.1 \pm 0.4$ & $1.5 \pm 0.5$ & $1.8 \pm 0.6$ & $<0.001$ \\
\hline eGFR, $\mathrm{mL} / \mathrm{min} / 1.73 \mathrm{~m}^{2}$ & $64 \pm 22$ & $48 \pm 19$ & $39 \pm 16$ & $<0.001$ \\
\hline Hemoglobin, g/dL & $11.2 \pm 0.6$ & $11.0 \pm 0.7$ & $10.9 \pm 0.7$ & $<0.001$ \\
\hline Transferrin saturation, $\%$ & $27.5 \pm 10.6$ & $27.1 \pm 10.8$ & $26.4 \pm 11.2$ & 0.007 \\
\hline Iron, $\mu \mathrm{g} / \mathrm{dL}$ & $80.4 \pm 34.5$ & $75.7 \pm 38.5$ & $74.3 \pm 37.3$ & 0.019 \\
\hline Ferritin, $\mu \mathrm{g} / \mathrm{L}$ & $116 \pm 133$ & $165 \pm 174$ & $179 \pm 190$ & $<0.001$ \\
\hline Platelets, $\times 10^{9} / \mathrm{L}$ & $251 \pm 80$ & $231 \pm 80$ & $212 \pm 73$ & $<0.001$ \\
\hline $\mathrm{WBC}, \times 10^{9} / \mathrm{L}$ & $6.5 \pm 2.1$ & $6.9 \pm 2.2$ & $6.7 \pm 2.2$ & 0.143 \\
\hline hsCRP, mg/dL & $2.4(1.1,5.4)$ & $2.7(1.1,7.2)$ & $3.0(1.3,7.2)$ & $<0.001$ \\
\hline NT-proBNP, pmol/L & $994(220,2334)$ & $1823(762,3820)$ & $2983(1196,7002)$ & $<0.001$ \\
\hline hsTnT, ng/ml & $12(9,17)$ & $28(21,34)$ & $46(33,71)$ & $<0.001$ \\
\hline
\end{tabular}

Patient characteristics are given as mean \pm SD for continuous variables and $\%$ of cases for categorical variables

$A C E$ angiotensin-converting enzyme, $A R B$ angiotensin receptor blocker, $B M I$ body mass index, $B P$ blood pressure, $e G F R$ estimated glomerular filtration rate, $h S C R P$ high-sensitivity C-reactive protein, $h s T n T$ highsensitive troponin

\section{Association of change in GDF-15 levels and outcomes}

An increase in serum GDF-15 of $>15 \%$ during follow-up was associated with a higher incidence of the primary outcome following the second sampling (Table 3 ) adjusting for randomization (HR 1.39 [1.15-1.69] $p<0.002$ ) and multivariable (HR $1.68[1.38-2.06] p<0.001)$ analyses with a similar pattern for the secondary composite and mortality outcomes (HR's of 1.40-1.73 after full adjustment (Table 3). Furthermore, adding change in GDF-15 to the fully adjusted model improved the C-statistics (all $p<0.004$ ) and a significant effect on NRI (all $p<0.001$ ) was observed for all endpoints (Table 3 ).

Supplemental Table 3 shows the association between change in GDF-15 and outcomes evaluated as tertiles. This analysis supports that those with the largest increase in GFD-15 (T3) had a higher incidence of all outcome measures. The mid tertile (T2) displayed a lower risk compared to the lower tertile. Baseline GDF-15 levels were strongly correlated with levels at 6 months $(r=0.83, p<0.001)$, but negatively correlated with change scores $(r=-0.23$, $p<0.001)$. Finally, we made a heatmap of the incidence 
Fig. 1 Association between baseline GDF-15 levels and the primary endpoint in the RED-HF cohort $(n=1582)$ during the whole study (mean follow-up 28 months, range 0.03-72.4 months) expressed as A restricted cubic spline with tertile cut-offs at enrollment shown as dotted lines and $\mathbf{B}$ Kaplan-Meier curves showing the cumulative incidence of the primary endpoint according to tertiles at enrollment

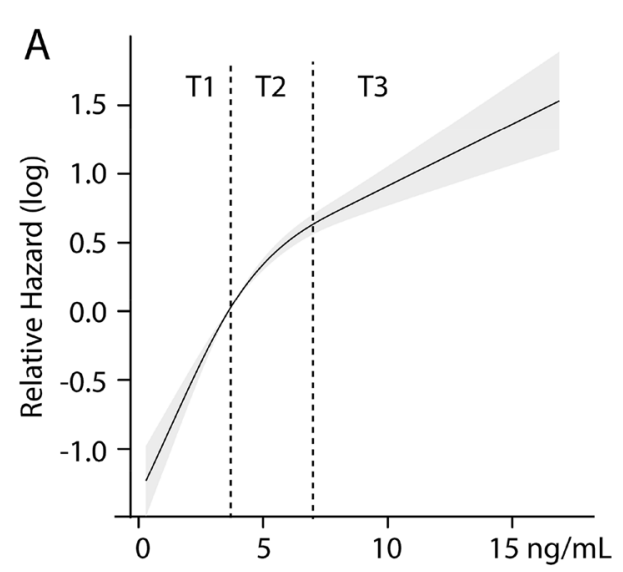

Table 2 Association of baseline GDF-15 with outcomes

\begin{tabular}{|c|c|c|c|c|c|}
\hline & Univariable & Step 1 & Step 2 & $\Delta \mathrm{C}$-index $(p \text { value })^{*}$ & NRI ( $p$ value $)^{*}$ \\
\hline \multicolumn{6}{|c|}{ All-cause mortality or first hospitalization for worsening heart failure, $n=798$} \\
\hline $\mathrm{T} 2$ & $2.10(1.72-2.57)$ & $1.63(1.32-2.01)$ & $1.20(0.96-1.49)$ & & \\
\hline $\mathrm{T} 3$ & $4.05(3.35-4.9)$ & $2.57(2.05-3.23)$ & $1.56(1.23-1.98)$ & & \\
\hline Continuous & $2.29(2.06-2.54)$ & $1.80(1.58-2.04)$ & $1.26(1.10-1.45)$ & & \\
\hline p-trend/p-cont* & $<0.001 /<0.001$ & $<0.001 /<0.001$ & $<0.001 / 0.001$ & $0.003(0.148)$ & $0.183(<0.001)$ \\
\hline \multicolumn{6}{|c|}{ Cardiovascular mortality or first hospitalization for worsening heart failure, $n=716$} \\
\hline $\mathrm{T} 2$ & $2.10(1.71-2.59)$ & $1.62(1.30-2.02)$ & $1.20(0.95-1.50)$ & & \\
\hline $\mathrm{T} 3$ & $3.94(3.22-4.81)$ & $2.47(1.94-3.13)$ & $1.56(1.23-1.99)$ & & \\
\hline Continuous & $2.25(2.01-2.51)$ & $1.74(1.52-1.98)$ & $1.22(1.05-1.41)$ & & \\
\hline $\mathrm{p}$-trend/p-cont* & $<0.001 /<0.001$ & $<0.001 /<0.001$ & $0.004 / 0.010$ & $0.002(0.212)$ & $0.150(0.003)$ \\
\hline \multicolumn{6}{|c|}{ All-cause mortality, $n=649$} \\
\hline $\mathrm{T} 2$ & $2.22(1.77-2.78)$ & $1.75(1.39-2.22)$ & $1.32(1.03-1.68)$ & & \\
\hline $\mathrm{T} 3$ & $3.93(3.17-4.88)$ & $2.62(2.03-3.38)$ & $1.64(1.25-2.14)$ & & \\
\hline Continuous & $2.32(2.05-2.59)$ & $1.89(1.64-2.18)$ & $1.37(1.17-1.60)$ & & \\
\hline $\mathrm{p}$-trend/p-cont* & $<0.001 /<0.001$ & $<0.001 /<0.001$ & $0.001 /<0.001$ & $0.005(0.108)$ & $0.166(0.001)$ \\
\hline \multicolumn{6}{|c|}{ Cardiovascular mortality, $n=543$} \\
\hline $\mathrm{T} 2$ & $2.31(1.81-2.94)$ & $1.82(1.40-2.35)$ & $1.35(1.04-1.77)$ & & \\
\hline $\mathrm{T} 3$ & $3.75(2.96-4.74)$ & $2.47(1.86-3.26)$ & $1.50(1.11-2.01)$ & & \\
\hline Continuous & $2.24(1.97-2.55)$ & $1.82(1.56-2.13)$ & $1.29(1.08-1.53)$ & & \\
\hline $\mathrm{p}$-trend/p-cont & $<0.001 /<0.001$ & $<0.001 /<0.001$ & $0.026 / 0.005$ & $0.003(0.220)$ & $0.099(0.061)$ \\
\hline
\end{tabular}

Hazard ratios and 95\% confidence interval are shown for tertile 2 and 3 and for GDF-15 as a continuous (log) variable in univariate (UNI) analysis, when adjusted for clinical and biochemical variables (Step 1), and last for CRP, TnT and NT-proBNP (Step 2)

*Comparing the fully adjusted models with and without inclusion of log GDF15

of the primary outcome according to quartiles of baseline and change in GDF-15. As shown in Fig. 2, the incidence was highest among all quartiles of change for quartile 4 of baseline GDF-15 with the highest incidence for quartile 4 of both baseline and change values. However, a markedly higher incidence for change in quartiles 3 and 4 vs. 1 and 2 was noted within quartile 1 of baseline GDF-15 (i.e. $28 \%$ vs. $20 \%$ ).

\section{GDF-15 and iron metabolism}

To understand the potential role of GDF-15 in iron metabolism and erythropoiesis, we further analyzed associations between GDF-15 and different markers related to iron metabolism. As shown in supplemental Table 4 and Fig. 3A, at baseline GDF-15 was inversely correlated with hemoglobin and positively with ferritin, with weaker positive associations with transferrin saturation and iron. In the 
Table 3 Association of change in GDF-15 with outcomes

\begin{tabular}{|c|c|c|c|c|c|}
\hline & Univariable & Step 1 & Step 2 & $\Delta \mathrm{C}$-index $(p \text { value })^{*}$ & NRI ( $p$ value $)^{*}$ \\
\hline \multicolumn{6}{|c|}{ All-cause mortality or first hospitalization for worsening heart failure } \\
\hline$\leq-15 \%$ & $1.06(0.86-1.29)$ & $0.95(0.77-1.17)$ & $0.98(0.80-1.21)$ & & \\
\hline$>15 \%$ & $1.39(1.15-1.69)$ & $1.65(1.36-2.01)$ & $1.68(1.38-2.06)$ & & \\
\hline p-trend & 0.002 & $<0.001$ & $<0.001$ & $0.050(<0.001)$ & $0.266(<0.001)$ \\
\hline \multicolumn{6}{|c|}{ Cardiovascular mortality or first hospitalization for worsening heart failure } \\
\hline$\leq-15 \%$ & $1.09(0.88-1.35)$ & $0.97(0.78-1.20)$ & $1.00(0.80-1.25)$ & & \\
\hline$>15 \%$ & $1.46(1.19-1.80)$ & $1.72(1.40-2.11)$ & $1.73(1.40-2.14)$ & & \\
\hline $\mathrm{p}$-trend & 0.001 & $<0.001$ & $<0.001$ & $0.051(<0.001)$ & $0.228(<0.001)$ \\
\hline \multicolumn{6}{|c|}{ All-cause mortality } \\
\hline$\leq-15 \%$ & $1.00(0.80-1.25)$ & $0.98(0.78-1.24)$ & $1.02(0.81-1.29)$ & & \\
\hline$>15 \%$ & $1.23(0.99-1.53)$ & $1.47(1.18-1.83)$ & $1.40(1.12-1.76)$ & & \\
\hline $\mathrm{p}$-trend & 0.118 & 0.001 & 0.007 & $0.038(0.004)$ & $0.219(<0.001)$ \\
\hline \multicolumn{6}{|c|}{ Cardiovascular mortality } \\
\hline$\leq-15 \%$ & $1.05(0.82-1.35)$ & $1.03(0.80-1.33)$ & $1.08(0.84-1.39)$ & & \\
\hline$>15 \%$ & $1.35(1.07-1.71)$ & $1.59(1.25-2.03)$ & $1.49(1.16-1.91)$ & & \\
\hline p-trend & 0.037 & $<0.001$ & 0.005 & $0.047(0.002)$ & $0.222(<0.001)$ \\
\hline
\end{tabular}

Hazard ratios and $95 \%$ confidence interval are shown for $\leq-15 \%$ and $>15 \%$ change vs. no change $(-15-15 \%)$ in univariate (UNI) analysis, when adjusted for clinical and biochemical variables (Step 1), and last for CRP, TnT and NT-proBNP (Step 2)

*Comparing the fully adjusted models with and without inclusion of log GDF15

Fig. 2 Heatmap showing association between baseline GDF-15 and change in GDF-15 on incidence $(\%)$ of the primary outcome. Both baseline (ng/mL) and change (\%) are shown as quartiles with limits

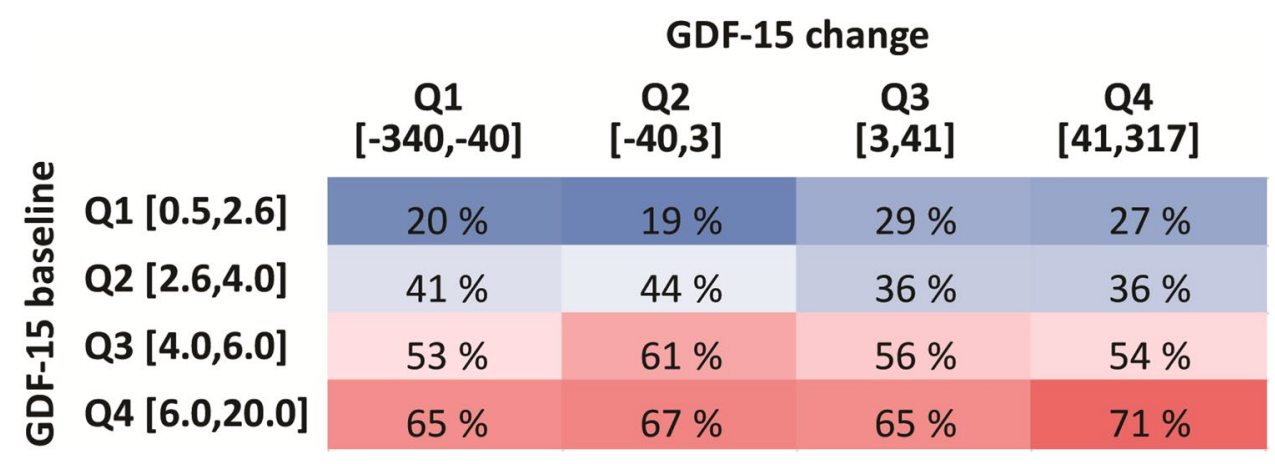

population as a whole, change in GDF-15 did not correlate with the iron parameters. At 6 months' follow-up, somewhat stronger associations between GDF-15 and markers of iron metabolism were observed, with no significant differences according to treatment group.

\section{Interactions between iron deficiency markers, GDF-15 and outcomes}

We next analyzed interactions between GDF-15 and iron status on outcome by dividing the iron status markers in tertiles and evaluating the prognostic role of GDF-15 (continuous log transformed) within each tertile. These data are presented adjusted for randomized treatment and after full multivariable adjustment in Supplemental Table 5 and graphically in Fig. 3B. The association between GDF15 and the primary outcome was not dependent on iron levels, while a stronger association was observed with increasing ferritin and transferrin saturation. Conversely, lower and mid-tertile levels of hemoglobin were associated with adverse outcomes with increasing GDF-15. These associations were quite similar for the secondary endpoints, but low or intermediate levels of iron were more strongly associated with worse outcome for the mortality endpoints (Supplemental Table 5).

\section{Effect of darbepoetin on GDF-15}

Plasma GDF-15 levels were similar at baseline in the two treatment groups (mean \pm SD $4866 \pm 3123 \mathrm{ng} / \mathrm{L}$ vs. $4781 \pm 3077 \mathrm{ng} / \mathrm{L}(p=0.79)$, placebo and Darbepoetin, respectively. During the course of the study, a small decrease in GDF-15 was observed in the Darbepoetin group $(p=0.032$, Fig. 3C), but not in the placebo group, yielding a modest but significant difference in relative change between the treatment 
A

\begin{tabular}{lrrr} 
& Baseline & Change & $1-$ year \\
\hline Iron & $-0.06^{*}$ & 0.01 & $-0.14^{* * *}$ \\
Ferritin & $0.14^{* * *}$ & 0.00 & $0.14^{* * *}$ \\
TS\% & $-0.06^{*}$ & 0.00 & $-0.10^{* *}$ \\
$\mathrm{Hgb}$ & $-0.21^{* * *}$ & 0.02 & $-0.24^{* *}$
\end{tabular}

C

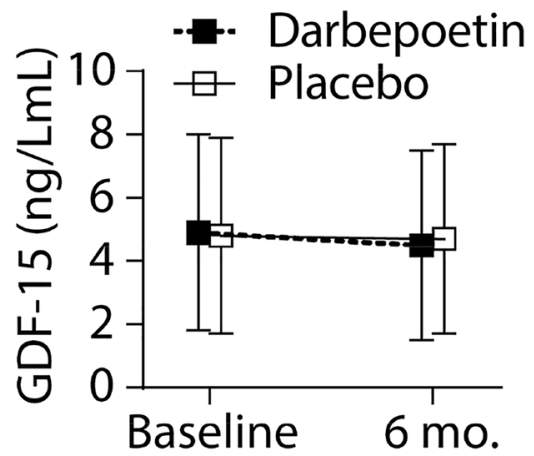

\section{B}

Fig. 3 Association between GDF-15 and iron status in HF patients. A Correlation between GDF-15 and iron status markers at baseline and 1 year and change between these time-points. B Association between GDF-15 and the primary endpoint (death from any cause or first hospitalization for worsening of HF), secondary endpoint (death

groups (mean change 0.0 vs. $0.1 p=0.039$, placebo and Darbepoetin, respectively).

\section{Interactions between darbepoetin alpha treatment and GDF-15 and outcomes}

We next evaluated whether baseline levels or change in GDF15 could identify patients who could benefit from Darbepoetin treatment. We found no evidence for this for baseline GDF15 , with interaction $p$ values for treatment*GDF-15 (tertiles) between 0.55 and 0.83 in unadjusted analysis for the different outcomes. A similar pattern was seen for change in GDF15 (as categorized above) with interaction $p$ values ranging between 0.11 and 0.82 .

\section{GDF-15 and unresponsiveness to darbepoetin in HF patients}

The median initial hemoglobin change in non-responders $(n=198)$ was $-0.25 \mathrm{~g} / \mathrm{dL}$ and $+1.00 \mathrm{~g} / \mathrm{dL}$ in the remainder of patients $(n=592)$. ROC analysis indicated no association between GDF-15 levels and unresponsiveness to ESA $(\mathrm{AUC}=0.51)$. Evaluated as a continuous variable, GDF-15 did not identify non-responders: HR 0.99 (0.81-1.18) and the HRs were unmodified by the addition of markers of iron metabolism to the model (i.e. ferritin, hemoglobin, transferrin saturation, iron). Similar results were obtained when GDF-15 was evaluated according to tertiles:tertile 2 HR 0.86 (95\% CI: 0.54-1.35); tertile 3 HR 0.85 (0.53-1.34). These 
HRs were unmodified by the addition of markers of iron metabolism to the model.

\section{Discussion}

In our study, higher baseline GDF-15 levels or an increase in GDF-15 levels during follow-up were associated with poor prognosis in adjusted analysis including TnT, CRP and NTproBNP, all strong predictors of outcome in HF patients [22, 23]. Furthermore, change in GDF-15 improved the discrimination and the magnitude of net improvement in sensitivity and specificity when added to these models. GDF-15 was associated with all iron status markers with the strongest correlation with hemoglobin but could not identify unresponsiveness or responsiveness to Darbepoetin. Our findings further support a role for GDF-15 as a strong and independent prognostic marker in patients with HF.

GDF-15 has been reported to add prognostic information in several CV disorders including myocardial infarction (MI), atherosclerosis, aortic stenosis, pulmonary hypertension and ischemic stroke $[6,24]$ as well as in HFrEF $[12-16,25]$ and more recently also in HF with preserved $\mathrm{EF}(\mathrm{HFpEF})[13,15,25]$. However, like several other inflammatory markers, GDF-15 is not specific for HF or other CV disorders and is not useful as a diagnostic tool, although capable of giving prognostic information as we have shown. Kempf et al. showed that in 455 patients with systolic HF, GDF-15 predicted total mortality independent of biochemical and clinical variables including NT-proBNP [12]. Chan et al. found that in 730 patients with systolic HF, baseline GDF-15 was associated with the composite outcome of allcause mortality and first re-hospitalization of HF also after adjusting for established clinical and biochemical variables including TnT and NT-proBNP, with a similar pattern in HFpEF [13]. Gaggin et al. found that in 151 patients with chronic HF, GDF-15 together with TnT and the soluble version of the interleukin 1 receptor member ST2 was independently associated with $\mathrm{CV}$ events also after adjusting for NT-proBNP [14]. In a substudy of Val-HeFT ( $n=1734)$, baseline GDF-15 levels were associated with all-cause mortality and the first morbid event after adjusting for clinical and biochemical variables [16]; the association remained significant for all-cause mortality but not for the first morbid event when further adjustments were made for TnT, CRP and NT-proBNP. Similar to the Val-HeFT study, we found that an increase in GDF-15 was strongly associated with poor prognosis, with significant improvement in discrimination analysis for all outcomes. However, the negative correlation between baseline GDF-15 and change in GDF-15, and relatively larger increase in incidence of the primary outcome with increasing quartiles of change within quartile 1 of baseline GDF-15, suggests patients with the largest increase are not only those presenting with high GDF-15 levels. Indeed, thus, serial analysis could be beneficial in identifying patients not detected by the initial measurement.

The independent association between GDF-15 and adverse outcome in chronic HF may have several non-mutually exclusive explanations. First, GDF-15 is strongly upregulated in the myocardium during wall stress and ischemia $[7,8]$, and it is possible that the plasma levels in HFrEF at least partly reflect up-regulation of GDF-15 in failing myocardium. Second, GDF-15 seems to reflect activation of inflammatory, myocardial remodeling and apoptotic pathways-, and the ability of a measure like GDF-15 to mirror several pathological processes is an important feature of a robust biomarker. Notably, GDF-15 exhibits potent antiinflammatory, anti-hypertrophic and anti-apoptotic properties and its up-regulation during HF seems to reflect multiple and counteractive mechanisms [7,8].

While the RED-HF trial failed to detect a benefit of an erythropoiesis-stimulating agent on clinical outcomes, this does not exclude the possibility that some individuals may benefit from correction of anemia. Enhanced hepcidin production has been implicated in the pathogenesis of anemia in chronic inflammatory disorders like HF [26], and GDF15 was recently identified as a hepcidin-suppression factor [11]. However, the suppressive effect of GDF-15 on hepcidin was not tested in this study and has not been reported in HF patients in other studies. Nor has an interaction between GDF-15 and erythropoiesis been clarified to our knowledge. In the present study, we found mostly weak correlations between GDF-15 and markers of iron metabolism. However, a stronger association between GDF15 and the primary outcome was observed with increasing ferritin and transferrin saturation, and conversely, lower and mid-tertile levels of hemoglobin were associated with adverse outcomes with increasing GDF-15, suggesting some interaction between GDF-15 and iron metabolism. Moreover, GDF-15 levels in our study population seems to be higher than in previous study in HF patients [9]. Although the GDF-15 assay was not calibrated against a universal standard, it is possible that the additional burden of anemia could have contributed to the elevated levels GDF-15 levels in the present study as compared to HF patients without anemia as also has been suggested by others [10].

\section{Limitations}

The strengths of the present investigation include the large number of patients studied with a high event rate, longitudinal sampling and adjustment for multiple relevant confounders. On the other hand, a randomized trial may not necessarily reflect the "real-world" HF population and the use of composite endpoints has an inborn limitation. Our 
patients also had anemia, and our results will, therefore, not apply to all chronic HF cohorts. However, anemia is common in HF, in particular in advanced cases. Finally, the lack of data on hepcidin levels is also a limitation of the present study.

\section{Conclusions}

Our findings indicate that GDF-15 is a promising prognostic marker in patients with chronic HF and anemia, with correlation to indices of iron metabolism. Future studies should examine whether this marker could be used for treatment-related decision-making and risk stratification in these patients.

Supplementary Information The online version contains supplementary material available at https://doi.org/10.1007/s00392-021-01944-6.

Author contributions All authors have made substantial contributions to the conception and design of the study, or acquisition of data, or analysis and interpretation of data, drafting the article or revising it critically for important intellectual content, and approved the final manuscript.

Funding Open access funding provided by University of Oslo (incl Oslo University Hospital). None.

Availability of data and materials The data that support the findings of this study are available from Amgen but restrictions apply to the availability of these data, which were used under license for the current study, and so are not publicly available. Data are, however, available from the authors upon reasonable request and with permission of Amgen.

Code availability Not applicable.

\section{Declarations}

Conflict of interest I.S.A., D.J.v.V., J.B.Y., J.J.V.M.: members of the RED-HF Executive Committee (no payments in the last 12 months). M.P.: member of the RED-HF Clinical Endpoint Committee and Executive Committee (no payments in the last 12 months); grants from Novartis and Sanofi; consulting fees from AstraZeneca, Bayer, Boehringer Ingelheim, DalCor, Gilead, GalaxoSmithKline, Janssen, Lilly USA, The Medicines Company, Merck, Novartis, Novo Nordisk, Relypsa, Sanofi, Thrasos, Genzyme and Teva; The Brigham and Women's Hospital has patents for the use of inhibitors of the renin-angiotensin system in selected survivors of myocardial infarction with Novartis. K.S.: consulting fees from AstraZeneca, Amgen, Novartis, Servier, Vifor Pharma; member of the RED-HF Executive Committee (no payments in the past 12 months). A.S.D.: grant to the institution to support endpoint adjudication activities from Amgen; grant from Novartis; consulting fees from Novartis, St Jude/Abbott, Relypsa, Janssen, AstraZeneca, Sanofi, Cheetah Medical. J.J.V.M.: consulting fees from Cytokinetics/Amgen; grant from Amgen. All other authors have no conflict of interest.
Ethical approval The trial complied with the Declaration of Helsinki and was approved by the Ethics Committees of the participating hospitals.

Consent to participate All patients provided written informed consent.

Consent for publication The provided information is anonymous and does not expose the identity of the patient.

Open Access This article is licensed under a Creative Commons Attribution 4.0 International License, which permits use, sharing, adaptation, distribution and reproduction in any medium or format, as long as you give appropriate credit to the original author(s) and the source, provide a link to the Creative Commons licence, and indicate if changes were made. The images or other third party material in this article are included in the article's Creative Commons licence, unless indicated otherwise in a credit line to the material. If material is not included in the article's Creative Commons licence and your intended use is not permitted by statutory regulation or exceeds the permitted use, you will need to obtain permission directly from the copyright holder. To view a copy of this licence, visit http://creativecommons.org/licenses/by/4.0/.

\section{References}

1. Anand I, McMurray JJ, Whitmore J, Warren M, Pham A, McCamish MA, Burton PB (2004) Anemia and its relationship to clinical outcome in heart failure. Circulation 110(2):149-154. https://doi. org/10.1161/01.CIR.0000134279.79571.73

2. O'Meara E, Murphy C, McMurray JJ (2004) Anemia and heart failure. Curr Heart Fail Rep 1(4):176-182

3. Anand IS, Gupta P (2018) Anemia and iron deficiency in heart failure: current concepts and emerging therapies. Circulation 138(1):80-98. https://doi.org/10.1161/CIRCULATIONAHA.118. 030099

4. Westenbrink BD, Visser FW, Voors AA, Smilde TD, Lipsic E, Navis G, Hillege HL, van Gilst WH, van Veldhuisen DJ (2007) Anaemia in chronic heart failure is not only related to impaired renal perfusion and blunted erythropoietin production, but to fluid retention as well. Eur Heart J 28(2):166-171. https://doi.org/10. 1093/eurheartj/ehl419

5. von Haehling S, Ebner N, Evertz R, Ponikowski P, Anker SD (2019) Iron deficiency in heart failure: an overview. JACC Heart Fail 7(1):36-46. https://doi.org/10.1016/j.jchf.2018.07.015

6. Wollert KC, Kempf T (2012) Growth differentiation factor 15 in heart failure: an update. Curr Heart Fail Rep 9(4):337-345. https://doi.org/10.1007/s11897-012-0113-9

7. Kempf T, Eden M, Strelau J, Naguib M, Willenbockel C, Tongers J, Heineke J, Kotlarz D, Xu J, Molkentin JD, Niessen HW, Drexler $\mathrm{H}$, Wollert KC (2006) The transforming growth factor-beta superfamily member growth-differentiation factor- 15 protects the heart from ischemia/reperfusion injury. Circ Res 98(3):351-360. https:// doi.org/10.1161/01.RES.0000202805.73038.48

8. Xu J, Kimball TR, Lorenz JN, Brown DA, Bauskin AR, Klevitsky R, Hewett TE, Breit SN, Molkentin JD (2006) GDF15/MIC-1 functions as a protective and antihypertrophic factor released from the myocardium in association with SMAD protein activation. Circ Res 98(3):342-350. https://doi.org/10.1161/01.RES.00002 02804.84885.d0

9. Wollert KC, Kempf T, Wallentin L (2017) Growth differentiation factor 15 as a biomarker in cardiovascular disease. Clin Chem 63(1):140-151. https://doi.org/10.1373/clinchem.2016.255174

10. Przybylowski P, Wasilewski G, Bachorzewska-Gajewska H, Golabek K, Dobrzycki S, Malyszko J (2014) Growth differentiation 
factor 15 is related to anemia and iron metabolism in heart allograft recipients and patients with chronic heart failure. Transpl Proc 46(8):2852-2855. https://doi.org/10.1016/j.transproceed. 2014.09.040

11. Tanno T, Bhanu NV, Oneal PA, Goh SH, Staker P, Lee YT, Moroney JW, Reed CH, Luban NL, Wang RH, Eling TE, Childs R, Ganz T, Leitman SF, Fucharoen S, Miller JL (2007) High levels of GDF15 in thalassemia suppress expression of the iron regulatory protein hepcidin. Nat Med 13(9):1096-1101. https://doi.org/ $10.1038 / \mathrm{nm} 1629$

12. Kempf T, von Haehling S, Peter T, Allhoff T, Cicoira M, Doehner W, Ponikowski P, Filippatos GS, Rozentryt P, Drexler H, Anker SD, Wollert KC (2007) Prognostic utility of growth differentiation factor-15 in patients with chronic heart failure. J Am Coll Cardiol 50(11):1054-1060. https://doi.org/10.1016/j.jacc.2007.04.091

13. Chan MM, Santhanakrishnan R, Chong JP, Chen Z, Tai BC, Liew OW, Ng TP, Ling LH, Sim D, Leong KT, Yeo PS, Ong HY, Jaufeerally F, Wong RC, Chai P, Low AF, Richards AM, Lam CS (2016) Growth differentiation factor 15 in heart failure with preserved vs. reduced ejection fraction. Eur J Heart Fail 18(1):81-88. https://doi.org/10.1002/ejhf.431

14. Gaggin HK, Szymonifka J, Bhardwaj A, Belcher A, De Berardinis B, Motiwala S, Wang TJ, Januzzi JL Jr (2014) Head-to-head comparison of serial soluble ST2, growth differentiation factor- 15 , and highly-sensitive troponin $\mathrm{T}$ measurements in patients with chronic heart failure. JACC Heart Fail 2(1):65-72. https://doi.org/ 10.1016/j.jchf.2013.10.005

15. George M, Jena A, Srivatsan V, Muthukumar R, Dhandapani VE (2016) GDF 15-a novel biomarker in the offing for heart failure. Curr Cardiol Rev 12(1):37-46

16. Anand IS, Kempf T, Rector TS, Tapken H, Allhoff T, Jantzen F, Kuskowski M, Cohn JN, Drexler H, Wollert KC (2010) Serial measurement of growth-differentiation factor-15 in heart failure: relation to disease severity and prognosis in the Valsartan Heart Failure Trial. Circulation 122(14):1387-1395. https://doi.org/10. 1161/CIRCULATIONAHA.109.928846

17. Swedberg K, Young JB, Anand IS, Cheng S, Desai AS, Diaz R, Maggioni AP, McMurray JJ, O'Connor C, Pfeffer MA, Solomon SD, Sun Y, Tendera M, van Veldhuisen DJ, Committees R-H, Investigators R-H (2013) Treatment of anemia with darbepoetin alfa in systolic heart failure. N Engl J Med 368(13):1210-1219. https://doi.org/10.1056/NEJMoa1214865

18. McMurray JJ, Anand IS, Diaz R, Maggioni AP, O'Connor C, Pfeffer MA, Polu KR, Solomon SD, Sun Y, Swedberg K, Tendera M, van Veldhuisen DJ, Wasserman SM, Young JB, Committees R-H, Investigators (2009) Design of the reduction of events with Darbepoetin alfa in heart failure (RED-HF): a phase III, anaemia correction, morbidity-mortality trial. Eur J Heart Fail 11(8):795801. https://doi.org/10.1093/eurjhf/hfp098

19. McMurray JJ, Anand IS, Diaz R, Maggioni AP, O'Connor C, Pfeffer MA, Solomon SD, Tendera M, van Veldhuisen DJ, Albizem
M, Cheng S, Scarlata D, Swedberg K, Young JB, Investigators R-HC (2013) Baseline characteristics of patients in the reduction of events with Darbepoetin alfa in heart failure trial (RED-HF). Eur J Heart Fail 15(3):334-341. https://doi.org/10.1093/eurjhf/ hfs 204

20. van der Meer P, Grote Beverborg N, Pfeffer MA, Olson K, Anand IS, Westenbrink BD, McMurray JJV, Swedberg K, Young JB, Solomon SD, van Veldhuisen DJ (2018) Hyporesponsiveness to Darbepoetin Alfa in patients with heart failure and anemia in the RED-HF study (reduction of events by Darbepoetin Alfa in heart failure): clinical and prognostic associations. Circ Heart Fail 11(2):e004431. https://doi.org/10.1161/CIRCHEARTFAILURE. 117.004431

21. Masson S, Anand I, Favero C, Barlera S, Vago T, Bertocchi F, Maggioni AP, Tavazzi L, Tognoni G, Cohn JN, Latini R, Valsartan Heart Failure T, Gruppo Italiano per lo Studio della Sopravvivenza nell'Insufficienza Cardiaca-Heart Failure I (2012) Serial measurement of cardiac troponin $\mathrm{T}$ using a highly sensitive assay in patients with chronic heart failure: data from 2 large randomized clinical trials. Circulation 125(2):280-288. https://doi. org/10.1161/CIRCULATIONAHA.111.044149

22. Ferreira JP, Ouwerkerk W, Tromp J, Ng L, Dickstein K, Anker S, Filippatos G, Cleland JG, Metra M, van Veldhuisen DJ, Voors AA, Zannad F (2020) Cardiovascular and non-cardiovascular death distinction: the utility of troponin beyond $\mathrm{N}$-terminal pro-B-type natriuretic peptide. Findings from the BIOSTAT-CHF study. Eur J Heart Fail 22(1):81-89. https://doi.org/10.1002/ejhf.1654

23. Pellicori P, Zhang J, Cuthbert J, Urbinati A, Shah P, Kazmi S, Clark AL, Cleland JGF (2020) High-sensitivity C-reactive protein in chronic heart failure: patient characteristics, phenotypes, and mode of death. Cardiovasc Res 116(1):91-100. https://doi.org/10. 1093/cvr/cvz198

24. Xu X, Li Z, Gao W (2011) Growth differentiation factor 15 in cardiovascular diseases: from bench to bedside. Biomarkers 16(6):466-475. https://doi.org/10.3109/1354750X.2011.580006

25. Izumiya $\mathrm{Y}$, Hanatani $\mathrm{S}$, Kimura $\mathrm{Y}$, Takashio $\mathrm{S}$, Yamamoto E, Kusaka H, Tokitsu T, Rokutanda T, Araki S, Tsujita K, Tanaka T, Yamamuro M, Kojima S, Tayama S, Kaikita K, Hokimoto S, Ogawa H (2014) Growth differentiation factor-15 is a useful prognostic marker in patients with heart failure with preserved ejection fraction. Can J Cardiol 30(3):338-344. https://doi.org/10.1016/j. cjca.2013.12.010

26. van der Putten K, Jie KE, van den Broek D, Kraaijenhagen RJ, Laarakkers C, Swinkels DW, Braam B, Gaillard CA (2010) Hepcidin-25 is a marker of the response rather than resistance to exogenous erythropoietin in chronic kidney disease/chronic heart failure patients. Eur J Heart Fail 12(9):943-950. https://doi.org/10.1093/ eurjhf/hfq099

\section{Authors and Affiliations}

\section{Thor Ueland ${ }^{1,4,8}$ - Lars Gullestad ${ }^{2,4,5,6} \cdot$ Lei Kou $^{9} \cdot$ James B. Young ${ }^{9} \cdot$ Marc A. Pfeffer $^{10} \cdot$ Dirk Jan van Veldhuisen $^{13}$. Karl Swedberg ${ }^{14,15}$. John J. V. Mcmurray ${ }^{16}$ - Akshay S. Desai ${ }^{10} \cdot$ Inderjit S. Anand ${ }^{11,12}$ - Pål Aukrust ${ }^{1,3,4,7,8}$}

1 Research Institute of Internal Medicine, Oslo University Hospital, Rikshospitalet, Nydalen, P. B. 4950, 0424 Oslo, Norway

2 Department of Cardiology, Oslo University Hospital, Rikshospitalet, Oslo, Norway
3 Section of Clinical Immunology and Infectious Diseases, Oslo University Hospital, Rikshospitalet, Oslo, Norway

4 Faculty of Medicine, University of Oslo, Oslo, Norway

5 Center for Heart Failure Research, University of Oslo, Oslo, Norway 
6 K.G. Jebsen Cardiac Research Center, University of Oslo, Oslo, Norway

7 K. G. Jebsen Inflammation Research Center, University of Oslo, Oslo, Norway

8 K. G. Jebsen Thrombosis Research and Expertise Center, University of Troms $\varnothing$, Tromsö, Norway

9 Cleveland Clinic, Cleveland, OH, USA

10 Cardiovascular Division, Brigham and Women's Hospital, Boston, MA, USA

11 VA Medical Center, Minneapolis, MN, USA
12 University of Minnesota, Minneapolis, MN, USA

13 University Medical Center Groningen, University of Groningen, Groningen, The Netherlands

14 Sahlgrenska Academy, University of Gothenburg, Gothenburg, Sweden

15 National Heart and Lung Institute, Imperial College, London, UK

16 BHF Glasgow Cardiovascular Research Centre, University of Glasgow, Glasgow, UK 